axSpA, and -20 for patients with PsA; figure 1). Quality of life improved by -5 for patients with axSpA and PsA, and by -7 for patients with RA.

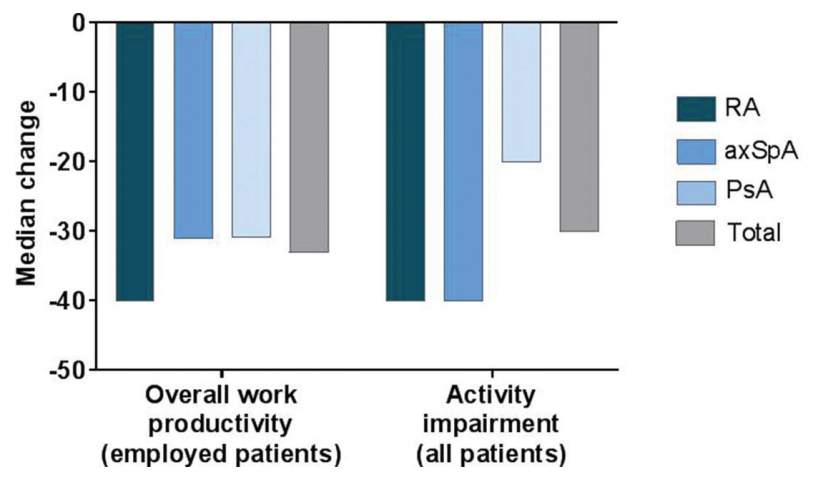

Abstract FRI0108 - Figure 1. WPAI questionnaire - Changes from baseline after 3 months of golimumab treatment

Conclusions: This interim analysis shows that golimumab treatment is effective in improving work productivity, daily activities as well as quality of life already within the first 3 months of treatment in RA, axSpA and PsA patients.

Disclosure of Interest: C. Dejaco Consultant for: Consulting fees from Merck Sharp and Dohme, Paid instructor for: Remuneration form Merck Sharp and Dohme, Speakers bureau: Merck Sharp and Dohme, T. Mueller: None declared, O. Zamani: None declared, U. Kurtz: None declared, S. Egger: None declared, J. Resch-Passini: None declared, A. Totzauer: None declared, W. Eisterer: None declared, B. Yazdani-Biuki: None declared, T. Schwingenschloegl: None declared, P. Peichl: None declared, A. Kraus: None declared, G. Naerr Employee of: Merck Sharp and Dohme Ges.m.b.H, V. Rickert Employee of: Merck Sharp and Dohme Ges.m.b.H

DOI: 10.1136/annrheumdis-2018-eular.2411

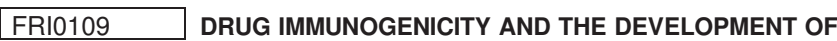 PARADOXICAL ADVERSE EVENTS WITH BIOLOGIC THERAPIES}

C. Ancuta $^{1,2}$, C. Pomirleanu ${ }^{1}$, R. Paiu ${ }^{1}$, G. Strugariu ${ }^{1}$, L. Petrariu ${ }^{1}$, E. Ancuta ${ }^{3}$, A. Chiriac ${ }^{4}$, R. Chirieac ${ }^{5} .{ }^{1}$ Clinical Rehabilitation Hospital; ${ }^{2}$ University of Medicine and Pharmacy Grigore T Popa lasi; ${ }^{3}$ Elena Doamna Clinical Hospital; ${ }^{4} \mathrm{CMI}$ Dermatology Nicolina Center, ${ }^{5}$ Sanocare Medical and REsearch Center, lasi, Romania

Background: Defined as de novo or exacerbation of pathologies that usually responds to biologic agents, paradoxical reactions encompass for a wide spectrum of manifestations (cutaneous, intestinal, ocular) reported during biological therapy (TNF and non-TNF medications) regardless of the underlying rheumatic or non-rheumatic disorder.

Objectives: To assess drug levels (DL) and anti-drug antibodies (ADA) in patients with paradoxical reactions (de novo or exacerbation of psoriasis, uveitis, Crohn's disease) induced by biological drugs (bDMARS) in Rheumatoid Arthritis (RA), Ankylosing Spondylitis (AS) and Psoriatic Arthritis (PsA).

Methods: We performed a retrospective observational study in cohorts of consecutive RA (267), PSA (65) and AS (145) undergoing TNF (i-TNF) or non-TNF biologics according to the local recommendations for initiation and monitoring, attending a single academic rheumatology department.

All patients were assessed at every 24 weeks months for disease activity and outcomes; supplementary, drug immunogenicity (ADA and DL) was systematically evaluated in patients developing paradoxical adverse events.

DL and ADA were measured with an ELISA assay and antibody binding test, respectively. Serum drug levels were considered positive for infliximab if $>0.035 \mu \mathrm{g} / \mathrm{mL}$, for adalimumab $>0.024 \mu \mathrm{g} / \mathrm{mL}$, for etanercept $>0.035 \mu \mathrm{g} / \mathrm{mL}$, rituximab $>0.75 \mu \mathrm{g} / \mathrm{mL}$, while the cut-offs for ADA positivity to infliximab was established at $5 \mathrm{AU} / \mathrm{ml}$, for adalimumab at $10 \mathrm{AU} / \mathrm{mL}$, etanercept at $142 \mathrm{AU} / \mathrm{mL}$, rituximab $140 \mathrm{AU} / \mathrm{mL}$ (ELISA, Progenika)

Results: 42 patients with paradoxical psoriasis ( 30 with de novo lesions, 18 with palmo-plantar pustulosis, 12 with exacerbation of pre-existent lesions), 12 with paradoxical uveitis ( 5 with a new onset uveitis) and 3 with paradoxical Crohn's were included in the final analysis. Paradoxical events related to abatacept, certolizumab (one de novo psoriasis per agent) or golimumab (2 new onset uveitis, one flare of Chron's disease) were excluded from the final analysis as no immunogenicity lab tests were provided.

Drug induced paradoxical events were described not only with all TNF inhibitors but also with rituximab. DL was in normal range in all these patients; in addition,
ADA were either negative, or, if present, had no impact on drug concentration. Furthermore, we found no significant differences in drug levels of TNF inhibitors for RA, PsA and AS.

Moreover, no statistical significant differences were observed in the detection of ADA between the three groups.

Conclusions: Patients who develop either true or borderline paradoxical $A E$ have adequate drug levels, with normal ADA concentrations.

Disclosure of Interest: None declared

DOI: 10.1136/annrheumdis-2018-eular.4854

\section{FRI0110 THE IMPACT OF SEROPOSITIVITY ON THE EFFECTIVENESS OF ABATACEPT VERSUS TNF INHIBITORS IN RHEUMATOID ARTHRITIS. REAL LIFE DATA FROM SEVERAL EUROPEAN REGISTRIES (THE PAN-ABA STUDY)}

D. Courvoisier ${ }^{1}$, D. Mongin ${ }^{1}$, M. Hetland ${ }^{2}$, K. Pavelka ${ }^{3,4}$, C. Turesson ${ }^{5}$, S.

A. Bergstra ${ }^{6}$, T. K. Kvien ${ }^{7}$, M. J. Santos ${ }^{8}$, V. Hernandez ${ }^{9}$, F. lannone ${ }^{10}$, J.

E. Gottenberg ${ }^{11}$, X. Mariette ${ }^{12}$, S. Kubo ${ }^{13}, Y$. Tanaka ${ }^{13}$, D. Choquette ${ }^{14}$,

R. Ionescu ${ }^{15}$, A. Finckh ${ }^{1} .{ }^{1}$ University Hospitals of Geneva, Geneva, Switzerland, ${ }^{2}$ DANBIO, Rigshospitalet, Denmark, ${ }^{3}$ Institute of Rheumatology, ${ }^{4}$ Rheumatology, First Faculty of Medicine, Charles U, Prague, Czech Republic, ${ }^{5}$ Lund University, Malmö, Sweden, ${ }^{6}$ LUMC, Leiden, Netherlands, ${ }^{7}$ Diakonhjemmet Hospital, Oslo, Norway, ${ }^{8}$ Reuma.pt, Lisbon, Portugal, ${ }^{9}$ Hospital Clinic, Barcelona, Spain,

${ }^{10}$ University of Bari, Bari, Italy, ${ }^{11}$ Chu, Strassbourg, ${ }^{12}$ Paris-Sud University, Le Kremlin-Bicêtre, France, ${ }^{13}$ University of Occupational and Environmental Health, Fukuoka, Japan, ${ }^{14}$ Institut de rhumatologie de Montreal, Chum, U de M, Montreal, Canada, ${ }^{15}$ Department of Internal Medicine and Rheumatology, Sf Maria Hospital, U Medicine and Pharmacy, Bucharest, Romania

Background: Rheumatoid factor (RF) and anti-citrullinated protein antibodies (ACPA) are used as diagnostic tools, but may also be used as prognostic factors, as these biomarkers have been associated with better clinical responses to some biologic agents in rheumatoid arthritis (RA).

Objectives: To compare the impact of seropositivity on drug discontinuation and effectiveness for abatacept (ABA) and TNF inhibitors (TNFi) in patients (pts) with RA.

Methods: Pooled analysis of 13 observational RA registries from countries (FR, IT, CZ, DK, NO, PT, RO, ES, SE, CH, NL, JP, CA) where both ABA and TNFi were available concomitantly. Inclusion criteria were RA diagnosis, treatment with $A B A$ or TNFi, and available RF or ACPA status. Main exposure was seropositivity: positive if RF or ACPA were positive, negative if both were negative, and missing if one was missing and the other was negative. Primary endpoint was drug discontinuation, analysed using Cox models, including treatment, seropositivity, and their interaction, adjusting for patient-, treatment-, and disease-characteristics using strata terms for country and calendar year. We first tested for effect modification by country by additionally including an interaction term between treatment, seropositivity and country. Since we found no effect modification, we took out the interaction term. Effectiveness was analysed using DAS28 remission and low disease activity (LDA) at 1 year, corrected for attrition using Lundex ${ }^{1}$.

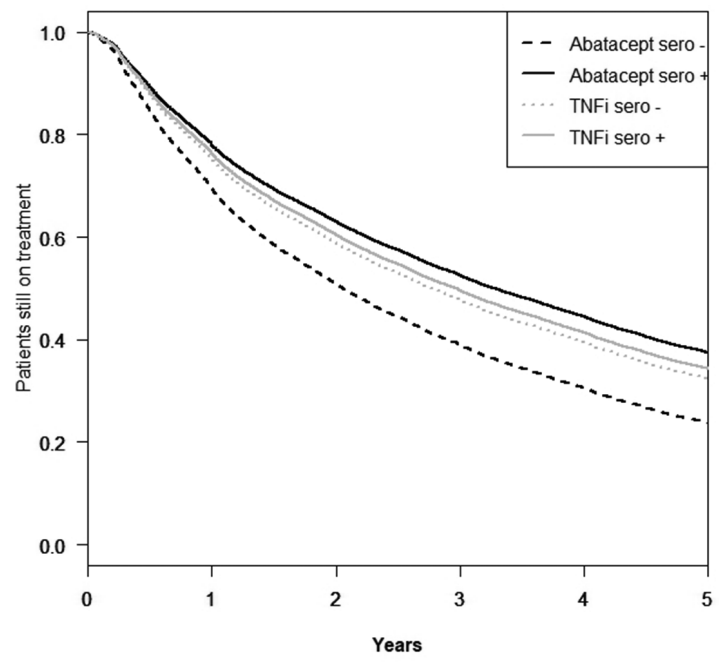

Results: Using data from 39266 treatment-courses, in crude analyses, seronegativity was associated with higher drug discontinuation for pts on ABA but not on TNFi ( $p$ interaction $<0.001$ ), with a hazard ratio $(\mathrm{HR})$ for seropositive vs seronegative of $0.74(95 \% \mathrm{Cl}: 0.66-0.82)$ for pts on $\mathrm{ABA}$ and $0.96(95 \% \mathrm{Cl}: 0.92-1.01)$ for 